\title{
Aprendizagem móvel e suas tecnologias: uma revisão sistemática da literatura
}

\author{
Karla Angélica Silva do Nascimento ${ }^{1}$, José Aires de Castro Filho ${ }^{2}$ \\ ${ }^{1}$ Departamento de Pós-Graduação em Educação Brasileira - CEP 60020-110 - \\ Universidade Federal do Ceará (UFC) - Fortaleza - CE - Brasil \\ ${ }^{2}$ Instituto UFC Virtual - CEP 60440-554 - Universidade Federal do Ceará (UFC) \\ Fortaleza - CE - Brasil \\ $\{$ karla, aires\} @virtual.ufc.br
}

\begin{abstract}
This paper presents empirical evidence of the use of mobile devices that support the teaching and learning process of teachers and students. Scientific survey from the following sources was admitted: Bank theses and journals of Higher Education Personnel Improvement Coordination (CAPES), in addition to digital libraries and scientific literature crawlers Scientific Electronic Library Online (SciELO), Education Resources Information Center (ERIC) and Science Direct. The results showed lack of research addressing mobile learning and its applications in elementary school, mostly.
\end{abstract}

Resumo. Este trabalho apresenta evidências empíricas da utilização de dispositivos móveis que auxiliam o processo de ensino e aprendizagem de professores e alunos. Admitiu-se o levantamento científico nas seguintes fontes: banco de teses e periódicos da Coordenação de Aperfeiçoamento de Pessoal de Nível Superior (CAPES), além das bibliotecas digitais e indexadores de literatura cientifica Scientific Electronic Library On-line (SciELO), Education Resources Information Center (ERIC) e Science Direct. Os resultados mostraram carência de pesquisas que abordem a aprendizagem móvel e seus aplicativos no Ensino Fundamental, principalmente.

\section{Introdução}

Uma série de publicações tem ampliado e proporcionado reflexões e discussões sobre a utilização de dispositivos móveis na educação [Barbour, et al., 2014; Roschelle, et al., 2010; Sharples, et al. 2010; Ting, 2013; Traxler, 2009]. As iniciativas de investigação têm verificado o potencial de dispositivos móveis na educação e, em particular, os benefícios de incorporá-los em sala de aula para desencadear a aprendizagem colaborativa e participação ativa do aluno. Estas tendências têm apresentado uma variedade de aplicações na aprendizagem móvel, aproveitando a mobilidade do usuário e de dispositivos para facilitar a aprendizagem em vários contextos, envolvendo diferentes espaços, tarefas e modos de interação.

Embora não exista uma definição específica para aprendizagem móvel, é importante entender que a mobilidade não é somente o movimento espacial, mas também as maneiras em que tal movimento pode permitir mudanças no tempo e demandar ações que minimizem fronteiras [Traxler, 2009].

No entanto, o ensino e a aprendizagem apoiados em tecnologias móveis colocam desafios para pesquisadores, profissionais de educação e técnicos de software na criação de ferramentas educacionais para fins didáticos claramente definidos que respondam às reais necessidades dos professores e alunos. Dessa forma, as experiências de aprendizagem que atravessam fronteiras espaciais, temporais e envolvem interações 
com tecnologias fixas e móveis, levaram professores e pesquisadores a refletirem sobre estratégias pedagógicas na utilização destes dispositivos para promover o ensino e a aprendizagem, por sua vez retratadas na literatura.

Nesse contexto, há uma nítida necessidade de se analisar a produção científica nacional e internacional sobre o processo aprendizagem móvel e a utilização de dispositivos móveis no Ensino Fundamental a partir de uma revisão sistemática da literatura. Segundo Keele (2007), esta revisão pode ser realizada por meio de dois elementos fundamentais: apontar lacunas existentes em uma determinada área de pesquisa ou prover uma coleção proeminente de trabalhos relacionados que podem apoiar novas pesquisas.

A revisão sistemática aqui realizada tem como questão principal: Qual o panorama atual das publicações científicas nacionais e internacionais sobre a aprendizagem móvel na prática colaborativa e a utilização de dispositivos móveis no Ensino Fundamental? Para respondê-la, foram definidos e analisados dois aspectos: 1) as metodologias e resultados abordados nos estudos sobre aprendizagem móvel na prática colaborativa; 2) os tipos de dispositivos móveis usados no ensino fundamental.

Portanto, o objetivo deste trabalho é apresentar evidências empíricas na utilização de dispositivos móveis que auxiliam o processo de ensino e aprendizagem de professores e alunos do Ensino Fundamental. Desta forma, buscou-se analisar pesquisas experimentais que tratam sobre as estratégias pedagógicas do uso de dispositivos móveis na escola, como também em aulas de campo.

\section{Procedimentos metodológicos}

A revisão sistemática é um tipo de investigação científica baseada em evidências, cujos resultados de pesquisas são coletados, categorizados, avaliados e sintetizados. Essa revisão é uma síntese rigorosa de todas as pesquisas relacionadas em uma questão particular. Ela é diferente da tradicional, já que ultrapassa possíveis vieses em todas as fases, seguindo uma metodologia rígida de busca e seleção de pesquisas; analisa a legitimidade do que foi encontrado; coleta, sintetiza e interpreta os dados provenientes das investigações (Rother, 2007).

Segundo Barros et al. (2008) a revisão sistemática tem as seguintes etapas: 1) selecionar todos os artigos científicos relevantes, relacionados a questão específica; 2) fazer a leitura dos resumos das publicações coletadas e eleger aquelas que satisfazem a critérios determinados; 3) citar os resultados dos artigos selecionados na etapa anterior por meio de tabelas; de modo opcional, 4) unificar os resultados individuais de cada publicação utilizando técnicas estatísticas complexas.

Frente ao exposto e buscando apresentar elementos que favoreçam reflexões para a aplicação de revisões sistemáticas no cenário da aprendizagem móvel na prática colaborativa e a utilização de dispositivos móveis no Ensino Fundamental, o presente item tem como objetivo apresentar as fases que compuseram a revisão sistemática deste estudo e os seus resultados.

A fim de selecionar pesquisas relacionadas ao objetivo central deste estudo, admitiu-se o levantamento científico nas seguintes fontes: banco de teses e periódicos da Coordenação de Aperfeiçoamento de Pessoal de Nível Superior (CAPES), além das 
bibliotecas digitais e indexadores de literatura científica Scientific Electronic Library On-line (SciELO), Education Resources Information Center (ERIC) e Science Direct.

Para os critérios de busca foram definidas palavras-chave que identificaram os estudos sobre aprendizagem móvel na prática colaborativa, assim, buscou-se as expressões "aprendizagem móvel", "prática colaborativa" e "aprendizagem colaborativa com suporte computacional (CSCL)", revisadas por pares com ajuda dos filtros para acompanhar, analisar e visualizar os estudos. $\mathrm{O}$ mesmo procedimento foi feito para os termos "dispositivos móveis", "tecnologias móveis" e "ensino fundamental". Estas palavras também foram traduzidas para o inglês e, consequentemente, agregadas à pesquisa.

Todas as ferramentas de buscas utilizadas possuem filtros que delimitam o assunto que se deseja avaliar: umas utilizam "and", "e", "ou", "or", outras possuem campos de data de publicação, tipo de material, idioma. Deste modo, foi realizada, em duas etapas, uma filtragem para a seleção dos artigos, dissertações e teses nacionais e internacionais com o objetivo de coletar maiores informações.

$\mathrm{Na}$ primeira etapa foi feita uma pré-seleção dos artigos pertinentes a questão principal, lendo os seus títulos, resumos e palavras-chave. Na segunda, todos os artigos pré-selecionados foram avaliados pela pesquisadora e a cada um deles foi identificado de que forma as palavras-chave foram reveladas, aplicadas e trabalhadas. Em seguida, os dados dos estudos proveram respostas à pergunta oriunda desta revisão sistemática. Os estudos que estavam em mais de uma base de dados diferente foram removidos para evitar duplicações. Por último, os dados dos artigos coletados foram sintetizados para apresentação dos resultados.

\section{Resultados}

A busca resultou em 108 artigos, publicados em revistas científicas: 20 são nacionais e 88 internacionais. Apesar de adicionar o termo "ensino fundamental" à pesquisa a fim de especificar melhor o estudo, todas as quatro ferramentas de busca compilaram também outros níveis e modalidades de ensino. De tal modo, verificou-se que 96 foram eliminados, porque se referiam ao Ensino Superior ou à Educação a Distância ou Educação Profissional ou não apresentavam um experimento sobre a utilização dos dispositivos móveis em práticas colaborativas, apresentado no gráfico seguinte.

\section{Gráfico 1 - Áreas de conhecimento encontradas na pesquisa}

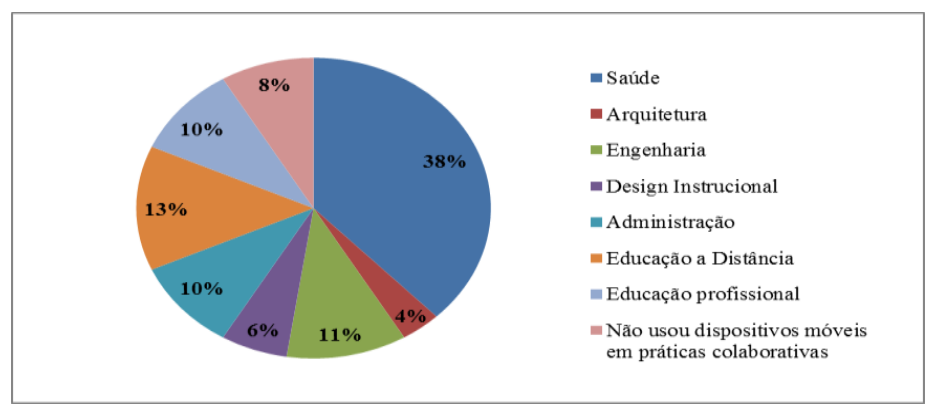

A Tabela 1 expõe o total de resultados encontrados em cada base de dados, bem como apresenta o quantitativo dos dados que são relevantes ao problema e às palavras-chaves citadas acima. 
CBIE-LACLO 2015

Anais dos Workshops do IV Congresso Brasileiro de Informática na Educação (CBIE 2015)

Tabela 1 - Quantitativo dos resultados nacionais e internacionais

\begin{tabular}{|c|c|c|c|c|}
\hline \multirow{2}{*}{ Base de dados } & \multicolumn{2}{|c|}{ Resultados encontrados } & \multicolumn{2}{|c|}{ Resultados relevantes } \\
\hline & Nacionais & Internacionais & Nacionais & Internacionais \\
\hline Periódicos CAPES & 14 & 23 & 0 & 7 \\
\hline ERIC & 0 & 4 & 0 & 4 \\
\hline SciELO & 6 & 7 & 0 & 0 \\
\hline Science Direct & 0 & 54 & 0 & 1 \\
\hline Total & 20 & 88 & 0 & 12 \\
\hline Total Geral & \multicolumn{2}{|c|}{108} & \multicolumn{2}{|c|}{12} \\
\hline
\end{tabular}

Após a leitura dos 12 artigos relevantes para esta pesquisa, percebeu-se que seus autores usaram, essencialmente, referências de estudos datados entre os anos de 2003 a 2009 com a finalidade de conceituar os termos aprendizagem móvel, aprendizagem colaborativa, dispositivos móveis e justificar uma teoria. No entanto, a análise dos textos selecionados está compreendida entre os anos de 2010 a 2015.

Em relação aos tipos de aplicativos móveis utilizados, percebe-se que boa parte são recursos fechados (Studywiz, FAO, Mobli21, MLE, TechPals), geralmente elaborados por grupos de pesquisa que ainda estão em processo de experiência. Outros são livres, mas requerem a criação de um cadastro para ser utilizado (Google Maps, Twitter, Flyer, Wikipédia). Alguns recursos já vem instalados nos próprios dispositivos móveis, tais como: GPS, áudio book, webcam.

\section{Tabela 2 - Recursos utilizados nos experimentos}

\begin{tabular}{|c|c|c|c|}
\hline Funcionalidade & $\begin{array}{l}\text { Dispositivo } \\
\text { móvel }\end{array}$ & $\begin{array}{l}\text { Recurso/ } \\
\text { Aplicativo }\end{array}$ & Atividade \\
\hline Produção de textos & Smartphone & Studywiz & $\begin{array}{l}\text { Produzir texto colaborativo com ajuda da plataforma de } \\
\text { aprendizagem Studywiz. }\end{array}$ \\
\hline $\begin{array}{l}\text { Representações } \\
\text { gráficas de frações }\end{array}$ & Palm Tops & FAO & $\begin{array}{l}\text { Identificar como se dá o apoio das tecnologias móveis durante } \\
\text { o desenvolvimento da atividade sobre frações. }\end{array}$ \\
\hline $\begin{array}{l}\text { Uso da Internet e o } \\
\text { ensino de língua } \\
\text { inglesa }\end{array}$ & Ipod Touch & $\begin{array}{l}\text { Áudio book } \\
\text { Google }\end{array}$ & $\begin{array}{l}\text { Apoiar a aprendizagem do conteúdo de línguas, ampliando o } \\
\text { tempo de pesquisa na Internet com atividades realizadas em } \\
\text { casa. }\end{array}$ \\
\hline $\begin{array}{l}\text { Compartilhamento } \\
\text { de imagens }\end{array}$ & Iphone & Flyer & $\begin{array}{l}\text { Solucionar os problemas expostos pelo professor por meio de } \\
\text { uma série de imagens em sequência (storyboard). }\end{array}$ \\
\hline $\begin{array}{l}\text { Registro das } \\
\text { localizações } \\
\text { espaciais }\end{array}$ & Iphone & $\begin{array}{l}\text { Webcam, } \\
\text { Google } \\
\text { Maps, GPS }\end{array}$ & $\begin{array}{l}\text { Promover a familiaridade dos alunos com o urbanismo e as } \\
\text { características sócio-geográfica dos diferentes distritos da } \\
\text { cidade de Duc de Montblanc de Rubí na Espanha. }\end{array}$ \\
\hline $\begin{array}{l}\text { Comunicação on- } \\
\text { line }\end{array}$ & Tablet & Twitter & $\begin{array}{l}\text { Explorar as habilidades de interpretação dos alunos no Museu, } \\
\text { bem como a compreensão sobre "Igualdade e crenças: os } \\
\text { direitos civis". }\end{array}$ \\
\hline Produção de textos & Tablet & Wikipédia & $\begin{array}{l}\text { Produzir textos sobre os espaços da escola onde cada grupo } \\
\text { deverá intervir na produção do outro. }\end{array}$ \\
\hline $\begin{array}{l}\text { Perguntas e respostas } \\
\text { sobre História } \\
\text { europeia }\end{array}$ & Blackberry & Mobl21 & $\begin{array}{l}\text { Projetar conteúdos de um curso em um aplicativo móvel e } \\
\text { examinar as percepções dos alunos em relação a aprendizagem. }\end{array}$ \\
\hline $\begin{array}{ll}\begin{array}{l}\text { Atividades } \\
\text { produção }\end{array} & \text { de } \\
\end{array}$ & Smartphone & MLE & $\begin{array}{l}\text { Desenvolver e organizar as atividades de produção realizadas } \\
\text { na sala de aula em vários formatos. }\end{array}$ \\
\hline Ensino de frações & Palm Tops & TechPals & $\begin{array}{l}\text { Solucionar os desafios mediados pelo docente, ajudando uns } \\
\text { aos outros a aprender sobre frações. }\end{array}$ \\
\hline
\end{tabular}

No Portal de Periódicos CAPES (http://www.periodicos.capes.gov.br/), sete artigos tratam os elementos pontuados neste estudo com alguma importância. Destes, dois discutem e analisam experiências de utilização de aparelhos celulares na escola. Norris e Soloway (2010) admitem que os alunos de uma escola localizada na zona rural de Ohio nos EUA, em um projeto realizado em 2008 e 2009, usaram celulares para 


\section{CBIE-LACLO 2015}

Anais dos Workshops do IV Congresso Brasileiro de Informática na Educação (CBIE 2015)

desenvolver $50 \%$ das suas atividades diárias na escola e, em seguida, usaram-nos para trabalhos fora do ambiente escolar. Os alunos utilizaram uma plataforma de aprendizagem chamada Studywiz que fornece alguns recursos digitais, entre eles um editor de texto colaborativo. Os resultados evidenciam que os estudantes do Ensino Fundamental mostraram melhores desempenhos na escrita. No entanto, a pesquisa não apresenta os procedimentos usados no trabalho colaborativo dos alunos, além disso, não estabeleceu situações que comprovassem a interação entre os alunos durante o projeto.

Em uma atividade colaborativa de matemática, um professor ajustou o currículo, seus horários de aula e procurou estratégias para identificar pares com frações complementares combinando setores circulares em uma turma de alunos do $4^{\circ}$ ano do Ensino Fundamental de uma escola em Cingapura na China, em seguida, formaram grupos entre eles. Para isso, utilizaram o aplicativo Form-A-One (FAO) que trata as frações na forma de representações gráficas. Boticki et al. (2011) investigaram e mapearam a colaboração de cada grupo com base em três aspectos: tecnológico, social e docente, a fim de identificar como se dá o apoio das tecnologias móveis durante o desenvolvimento da atividade. Segundo os autores, os suportes tecnológico e social eram recíprocos, os estudantes se comunicavam e negociavam verbalmente, como também por meio do dispositivo móvel. Além disso, essas tecnologias facilitaram na compreensão dos problemas mediados pelo professor e promoveram avanços nas resoluções dos problemas, pois os alunos visualizavam as frações dos outros, podendo se referir a seus dispositivos e percorrer as listas correspondentes, ajudando, explicando e justificando suas hipóteses. O professor foi capaz de especificar parâmetros na resolução dos problemas que impactaram diretamente na realização e nas possibilidades de colaboração dos alunos, ou seja, em cada etapa, o professor questionava, orientava os alunos em relação aos novos problemas, incentivava-os a percorrer a sala e a negociar.

Liu et al. (2014) investigaram uma iniciativa de aprendizagem móvel em um grande distrito escolar nos Estados Unidos a partir do fornecimento de dispositivos iPod touch durante todos os dias da semana em qualquer tempo e espaço para professores e alunos de língua inglesa. Os resultados revelaram que o iPod touch foi usado para apoiar a aprendizagem do conteúdo de línguas, fornecer apoio pedagógico diferenciado e ampliar o tempo de pesquisa com atividades realizadas em casa. No entanto, alguns desafios foram identificados, tais como: necessidade de formação profissional e pessoal de suporte técnico.

As aulas de campo com a utilização de dispositivos móveis foram destaques em três estudos. No primeiro, Laru et al. (2012) identificaram e compararam o desempenho de 22 alunos do $7^{\circ}$ ano do Ensino Fundamental, divididos em duas duplas e seis trios, durante discussões argumentativas com apoio de telefone celular, na situação 1:1, realizadas em uma viagem ao parque natural no norte da Finlândia. O projeto consistia em solucionar os problemas expostos pelo professor por meio de uma série de imagens em sequência (storyboard) por meio do aplicativo Flyer que permite criar uma rede social transmitindo e buscando informações de pessoas ou grupos. No projeto, os alunos apresentavam seus próprios argumentos aos questionamentos do professor na forma de mensagens e comparavam com os demais. Segundo os pesquisadores o uso da ferramenta de mensagens promoveu interações argumentativas durante os questionamentos dos alunos o que eu foi possível estabelecer níveis das respostas e da 


\section{CBIE-LACLO 2015}

Anais dos Workshops do IV Congresso Brasileiro de Informática na Educação (CBIE 2015)

participação e, consequentemente a criação de uma nova sequência de imagens para produção de um vídeo.

No segundo, Pérez-Sanagustín et al. (2012) recomendam que as atividades colaborativas com apoio dos dispositivos móveis nas aulas de campo devem considerar quatro fatores: o espaço, o método pedagógico, os participantes e a História. esses fatores compõem o termo 4SPPIces e estabelecem um quadro que produz um roteiro para facilitar o desenvolvimento dessas atividades. Os pesquisadores testaram o 4SPPIces em uma turma com 34 alunos e dois professores (titular e auxiliar). O roteiro teve o propósito de resolver as limitações de uma atividade, que ocorre todos os anos, de trabalho de campo na disciplina de Geografia para promover a familiaridade dos alunos com o urbanismo e as características sócio-geográfica dos diferentes distritos da cidade de Duc de Montblanc de Rubí na Espanha. Os autores revelaram que o fator espaço provou ser um bom mecanismo para utilizar ferramentas disponíveis (webcam, GPS, pesquisa de mapas) nas tecnologias móveis, devido o registro das localizações espaciais, onde ocorre a atividade. Devido à organização do projeto, os docentes se limitavam a observar e tirar dúvidas sobre o conteúdo e sua comunicação com os alunos era de forma presencial, pontual e verbal.

No terceiro, Charitonos et al. (2012) investigaram o uso de redes sociais e tecnologias móveis em uma excursão escolar ao Museu de Londres com 29 alunos do $9^{\circ}$ ano e o professor de História de uma escola de Milton Keynes, localizada a $72 \mathrm{~km}$ de Londres. O trabalho pedagógico em museus está ancorado nas diferentes perspectivas socioculturais de aprendizagem com foco na mediação de artefatos para compreensão do que se vê, lê e ouve. Neste sentido, o projeto teve como objetivo explorar as habilidades de interpretação dos alunos, bem como a compreensão sobre o conhecimento disciplinar, especificamente sobre "Igualdade e crenças: os direitos civis", desenvolvidos ao longo do tempo e materializados de várias formas (visual, escrita e oral) e meios de comunicação (aplicativos on-line). Os alunos foram divididos em oito grupos para coletar algumas evidências apresentadas em três galerias com o uso de tablet e do Twitter, para inserção de notas, fotos e postagens, a fim de criar quatro questões diferentes sobre o assunto e, posteriormente, na sala de aula, criar uma apresentação. Os autores concluíram que a utilização do Twitter melhorou a compreensão, participação e entusiasmo dos alunos durante a visita ao museu. As interações on-line ajudaram na negociação e na troca de conhecimento entre os estudantes. Além disso, as tecnologias não interromperam e nem perturbaram a autenticidade do cenário, pelo contrário, elas tiveram um impacto sobre a dinâmica social e pedagógica proposta na visita ao museu, como também, auxiliaram o processo de construção de significados de forma compartilhada. Contudo, o papel do professor na pesquisa ficou restrito em observar os alunos e ajudá-los em questões relacionadas à segurança no local, assim, não foi possível compreender a mediação docente e a ligação entre os conteúdos vistos em sala e na aula de campo.

Os três últimos estudos revelaram que os aplicativos e tecnologias móveis nas aulas de campo possibilitaram registros das informações coletadas nos ambientes externos à escola e foram importantes para o desenvolvimento de atividades de comparação, compartilhamento e produção, além de permitir continuidade dos conteúdos curriculares e promover debates sobre o que foi analisado e revelado. 
Ting (2013) realizou um estudo para identificar como os alunos do $6^{\circ}$ ano do Ensino Fundamental de uma escola de Taiwan na China, interagiam-se ao utilizar tecnologias móveis nas atividades escolares. Cinquenta e sete alunos divididos em dezenove grupos participaram deste experimento. Antes de iniciarem as atividades, eles preencheram um questionário sobre conhecimento prévio no uso de tecnologias móveis. $87 \%$ responderam que conheciam, mas não tinham usado, os outros $13 \%$ afirmaram ter utilizado em algum momento fora da escola. A atividade consistia em produzir textos sobre os espaços da escola e cada grupo deveria intervir na produção do outro. Após a atividade, cada participante escreveu um ensaio sobre a tecnologia móvel para medir seu desempenho de aprendizagem. Os resultados deram ênfase a visão dos alunos sobre a utilização de dispositivos móveis no dia a dia da escola e estes foram unânimes em dizer que estas tecnologias permitem melhor interação entre eles e tornam o aprendizado mais prático e repleto de possibilidades, pois possui diversos aplicativos que ajudam no desenvolvimento da atividade.

O ERIC (http://eric.ed.gov/) é um serviço de indexação de publicações na área de educação patrocinado pelo departamento de educação do governo americano. Ele indexa revistas internacionais de cunho educativo, anais de conferências, relatórios e produções bibliográficas. Desse acervo foram selecionados quatro artigos.

O primeiro estudo analisou a utilização de um aplicativo que funciona em dispositivos móveis, chamado Mobl21, por 11 estudantes com idade entre 10 a 12 anos matriculados em um curso suplementar de História Europeia, inicialmente, realizado em ambiente virtual de aprendizagem, oferecido em uma escola no Centro-Oeste dos EUA. Segundo Barbour et al. (2014), a implantação do Mobl21 no lugar do ambiente virtual do curso, na visão dos alunos, causou mudança pedagógica. Este aplicativo auxilia a aprendizagem de diversos temas educativos através de perguntas, apresentação de vídeos, textos e funciona em três sistemas operacionais: iOS, Android e Blackberry, bem como em uma versão emulada que pode ser executada em qualquer dispositivo móvel. Ao longo de quatro semanas usando Mobl21, os alunos acessaram o material didático e foram desafiados a responder questões sobre o conteúdo. Como resultado, as percepções dos alunos sobre Mobl21 foram justificadas pela usabilidade e mobilidade: 1) facilidade de acesso das ferramentas em qualquer dispositivo; 2) regularidade nos estudos, visto que a tecnologia está à mão; e, 3) pode ser utilizado em qualquer espaço e tempo. No entanto, o estudo revelou que os alunos tiveram dificuldades em se adaptar às funcionalidades do Mobl21 em seus próprios celulares e sentiram falta da mediação docente: quando tinham dúvidas sobre os conteúdos não podiam recorrer aos fóruns, pois o aplicativo não possuía tal recurso.

Já o segundo experimento, Norris, Hossain e Soloway (2011) apresentam os resultados de um estudo com estudantes do $4^{\circ}$ ano do Ensino Fundamental de uma escola de Cingapura, na China. Foi implantado um aplicativo chamado de Ambiente de Aprendizagem Móvel (Mobile Learning Environment-MLE) e permitiu que todas as atividades fossem expostas no smartphone. Por exemplo, na atividade sobre plantas, os alunos foram convidados a criar um mapa conceitual, uma animação, uma planilha eletrônica. A produção discente é representada no MLE. Neste projeto, os alunos tiveram acesso ao dispositivo de forma integral, ou seja, o smartphone foi usado em $100 \%$ do tempo de aula para desenvolver cada tarefa, bem como fora da escola. No entanto, o estudo mostra que a colaboração é uma habilidade que os professores de 


\section{CBIE-LACLO 2015}

Anais dos Workshops do IV Congresso Brasileiro de Informática na Educação (CBIE 2015)

Cingapura estão tentando trabalhar com seus alunos devido a necessidade de repensar o currículo para tirar o máximo proveito dos smartphones; implementar estratégias pedagógicas baseadas em indagações que apoiem as metas curriculares; e, acompanhar o impacto dessa mudança no desempenho dos alunos. Apesar disso, os autores presumem que a utilização de celulares nas escolas possui custo menor e preveem que dentro de cinco anos cada criança nos Estados Unidos vai usar um dispositivo e aplicativos de aprendizagem móvel. A investigação observou que a situação $1: 1$, se não for devidamente planejada, oferece pouca vantagem sobre os usos tradicionais da tecnologia.

Dois artigos não possuem experimentos, estes tratam sobre as previsões governamentais, na administração de Barack Obama, sobre um projeto que pretende colocar um dispositivo móvel nas mãos de todos os alunos do Ensino Fundamental e Médio. Com base nos estudos de Ash (2010) e Norris e Soloway (2015), as escolas dos EUA precisam melhorar a infraestrutura das salas de aula, as estratégias de avaliação, ensino, aprendizagem e produtividade para integrar as tecnologias móveis à prática pedagógica docente e à realidade dos alunos. Segundo Ash (2010), o plano, intitulado "Transformar a educação americana: aprendendo com a tecnologia", foi escrito ao longo de nove meses, por educadores e pesquisadores enfatiza a importância de alavancar a tecnologia para personalizar aprendizagem para cada aluno. Para Norris e Soloway (2015), o plano recomenda melhorias e ampliação do uso de currículos multimídia, avaliações formativas que fornecem atualizações regulares sobre o progresso dos alunos e formação de professores para usar os recursos digitais a fim de aprimorar o processo de ensino e de aprendizagem.

Science Direct (http://www.sciencedirect.com/) é uma plataforma on-line americana, que permite acesso a artigos em texto completo escritos por autores do cenário científico nas principais áreas do conhecimento. Dessa plataforma foi selecionada uma pesquisa sobre a utilização de uma ferramenta colaborativa móvel no ensino de frações, chamada Tecnologia Mediada de Aprendizagem Assistida por Pares (Technology-Mediated Peer-Assisted Learning, TechPals) ${ }^{1}$, para alunos do $4^{\circ}$ ano do Ensino Fundamental em uma escola de São Francisco, na Califórnia-EUA. Roschelle et al. (2010) investigaram que TechPals fornece evidências de que para desenvolver práticas colaborativas eficazes, é necessário ampliar o foco para além ferramentas. Ao utilizar TechPALS os alunos interagiam-se para solucionar os desafios, mediados pelo docente, ajudando uns aos outros a aprender sobre frações. Os autores reforçam que as atividades devem ser contextualizadas para integrar as ações de colaboração entre os alunos com o currículo. No entanto, a pesquisa demonstra uma experiência limitada em relação a colaboração entre pares. As notas de campo evidenciaram mais as dificuldades técnicas do que pedagógicas. Isso contribuiu para melhorar as funcionalidades do recurso em questão.

Após esse levantamento, nenhuma menciona o potencial da aprendizagem móvel na prática colaborativa para o desenvolvimento de atividades que vão além do ambiente escolar propriamente dito. Além disso, os dois estudos não analisaram os

\footnotetext{
${ }^{1}$ TechPALS foi desenvolvido pelo professor Miguel Nussbaum da Universidad Catolica de Santiago, Chile, como também implementado em escolas da Califórnia nos EUA. Tem como objetivo ensinar frações para estudantes do Ensino Fundamental.
} 
dispositivos móveis a partir do ponto de vista do ensino e do seu papel na aprendizagem. Mais importante ainda, o desenvolvimento e o uso de padrões de tecnologia estão mudando rapidamente, exigindo uma análise das tendências e funcionalidades dos diversos tipos de dispositivos móveis na educação, isso também não foi revelado nos estudos. Apesar disso, essas pesquisas relatam resultados positivos que mostram o envolvimento dos alunos, no entanto, é necessário verificar se o que ocorre durante o processo de aprendizagem móvel está sendo também trabalhado e relacionado ao contexto escolar que ultrapasse os muros da escola, ou seja, como o professor pode mediar práticas colaborativas com dispositivos móveis no contexto da sala de aula em situações presentes no dia a dia do aluno?

\section{Considerações finais}

A busca realizada nesta revisão sistemática da literatura resultou na pré-seleção de 108 artigos dos quais 12 foram incluídos para a extração de dados. A distribuição temporal mostrou que os artigos foram publicados entre os anos de 2010 a 2015. Além disso, de 2012 para 2013 houve um aumento na quantidade de artigos publicados na área de aprendizagem móvel para Ensino Fundamental.

A distribuição geográfica das instituições de pesquisa mostrou que a maior parte delas está localizada em países estrangeiros. Além disso, 3/4 das pesquisas que foram realizadas por vários grupos de maneira colaborativa.

A discrepância entre a quantidade de artigos publicados nos contextos do Ensino superior, Educação a Distância, Educação Profissional e, aqueles que não apresentavam um experimento sobre a utilização dos dispositivos móveis em práticas colaborativas, mostrou claramente a carência de pesquisas que abordem a aprendizagem móvel e seus aplicativos no Ensino Fundamental, principalmente.

A organização apresentada para os tipos de artefatos propostos sugere que há uma preferência nítida dos pesquisadores por construírem ferramentas que apoiem as tecnologias móveis em diversos contextos de aprendizagem.

\section{Referências}

ASH, Katie. (2010) US Ed-Tech Plan Urges Rethinking in K-12 Schools. Education Week, v. 29, n. 24, p. 1-16.

BARBOUR, Michael; GRZEBYK, Tamme Quinn; EYE, John. Any Time, Any Place, Any Pace-Really? (2014) Examining Mobile Learning in a Virtual School Environment. Turkish Online Journal of Distance Education-TOJDE, v. 15, n. 1.

BARROS, A. C.; WAINER, J.; CLAUDIO, K.; FERREIRA, L. R. R.; DWYER, T. (2008) Uso de computadores no Ensino Fundamental e Médio e seus resultados empíricos: uma revisão sistemática da literatura. Revista Brasileira de Informática na Educação. 16(1), p. 57-68.

BOTICKI, I.; LOOI, C. K.; WONG, L. H. (2011) Supporting Mobile Collaborative Activities through Scaffolded Flexible Grouping. Educational Technology \& Society, p. 190-202. 


\section{CBIE-LACLO 2015}

Anais dos Workshops do IV Congresso Brasileiro de Informática na Educação (CBIE 2015)

CHARITONOS, Koula; Blake, C., Scanlon, E.; Jones, A. (2012) Museum learning via social and mobile technologies: (How) can online interactions enhance the visitor experience? British Journal of Educational Technology, v. 43, n. 5, p. 802-819.

KEELE, Staffs. (2007) Guidelines for performing systematic literature reviews in software engineering. Technical report, EBSE Technical Report EBSE.

LARU, Jari; JÄRVELÄ, Sanna; CLARIANA, Roy B. Supporting collaborative inquiry during a biology field trip with mobile peer-to-peer tools for learning: a case study with K-12 learners. Interactive Learning Environments, v. 20, n. 2, p. 103-117, 2012.

LIU, M.; NAVARRETE, C. C.; WIVAGG, J. (2014) Potentials of Mobile Technology for K-12 Education: An Investigation of iPod touch Use for English Language Learners in the United States. Educational Technology \& Society, p. 115-12.

PÉREZ-SANAGUSTÍN, M.; SANTOS, P.; HERNÁNDEZ-LEO, D.; BLAT, J. (2012) 4SPPIces: A case study of factors in a scripted collaborative-learning blended course across spatial locations. International Journal of Computer-Supported Collaborative Learning, v. 7, n. 3, p. 443-465.

ROTHER, Edna T. (2007) Revisão sistemática versus revisão narrativa. Acta Paulista de Enfermagem, v. 20, n. 2, p. V-VI.

ROSCHELLE, J.; RAFANAN, K.; ESTRELLA, G.; NUSSBAUM, M.; CLARO, S. (2010) From handheld collaborative tool to effective classroom module: Embedding CSCL in a broader design framework. Computers \& Education, v. 55, n. 3, p. 10181026.

SHARPLES, M., ARNEDILLO-SÁNCHEZ, I., MILRAD, M.; VAVOULA, G. (2010) Mobile learning. Springer Netherlands, p. 233-249.

NORRIS, Cathleen A.; SOLOWAY, Elliot. (2015) Mobile Technology in 2020: Predictions and Implications for K-12 Education. Educational Technology, v55 n1 p12-19 Jan-Feb.

NORRIS, Cathleen; HOSSAIN, Akhlaq; SOLOWAY, Elliot. (2011) Using Smartphones as Essential Tools for Learning. Educational Technology, v. 51, n. 3, p. $18-25$.

NORRIS, Cathleen A.; SOLOWAY, Elliot. (2010) Innovative Leaders Take the Phone and Run: Profiles of Four Trailblazing Programs. District Administration, v. 46, n. 6, p. 35 .

TING, Yu-Liang. (2013) Using mobile technologies to create interwoven learning interactions: An intuitive design and its evaluation. Computers \& Education, v. 60, n. 1, p. 1-13.

TRAXLER, J. (2009) Current state of mobile learning. In ALLY, M. (Ed.), Mobile learning: Transforming the delivery of education and training. Edmonton: AU Press. p. 9-24. 